\title{
An update on prevention and treatment of osteoporosis
}

\author{
Shiva Borji, Hamid Nasri*
}

\begin{abstract}
Osteoporosis is an important skeletal disease in which, bone density slightly diminishes as long as the bone becomes porous like a sponge. This disorder happens because the skeleton is not able to replace old bones with new bones. Therefore bones become weaker, lose their strength and the risk of bone fractures (mostly spine and hip fractures) increases as individuals get older. Bone porosity, enhancement of bone length, reduction of bone size and livability of osteocytes are other factors that diminish bone strength. Patients with osteoporosis feel no signs unless their bone is broken after a fall or any other injury usually at age 50 and older. Spine fractures are scarcely reported and most of the time is invisible. This type of fracture can cause severe health problems like pain, curvature or flexure in your upper back and losing height that prevent you going away. Hip fractures are associated with mortality meanssome patients may die during difficulties related to this type of fractureor efforts to improve it. According to the latest research due to prevalence of osteoporosis, more than 200 million people in the world are suffering from osteoporotic fractures. Some different factors identified that contribute to high risk of osteoporosis and fractures. Thus recognizing the related these risk factor, will lead to take further steps to avoid developing osteoporosis and having bone's fractures. The main goal of this review is to make an aware on osteoporosis and some important factors to reduce the risk of developing osteoporosis.

Keywords: Osteoporosis, Hip fracture, Bone density, Vitamin D, Calcium

Please cite this paper as: Borji S, Nasri H. An update on prevention and treatment of osteoporosis. J Parathyr Dis. 2017;5(2):28-31. DOI: $10.15171 /$ jpd.2017.01.

Copyright @ 2017 The Author(s); Published by Nickan Research Institute. This is an open-access article distributed under the terms of the Creative Commons Attribution License, which permits unrestricted use, distribution, and reproduction in any medium, provided the original work is properly cited.
\end{abstract}

\section{Introduction}

As the age of the world's population gets more, the incidence and prevalence of osteoporosis increase further. Osteoporosis happens in both industrialized and developing countries. According to estimations, more than 200 million of women have osteoporosis worldwide. Osteoporosis is responsible for approximately the death of 37500 people each year after osteoporotic fractures. In the United States, osteoporosis threats 24 million individuals, approximately $80 \%$ is related to women and $20 \%$ is related to men. On the other hand, one in 4 women at age 50 years and more have osteoporosis and one in 8 men at age 50 years old and more have osteoporosis (1). Around 1.5 million and more fractures in women are caused by osteoporosis each year. More than half a million fractures are vertebral, 300000 are hip fractures, 200000 are wrist fractures, and 300000 fractures are related to other sites $(2,3)$.

\section{Materials and Methods}

In this review a variety of sources have been used by searching through PubMed/Medline, Scopus, EMBASE, EBSCO and directory of open access journals (DOAJ). The search was conducted using combination of the following key words and, or their equivalents; osteoporosis, hip fracture, bone density, vitamin $\mathrm{D}$, and calcium.

\section{Epidemiology}

The prevalence of osteoporosis rises progressively with age both in women and in men. According to estimations in the United States, the percentage of women diagnosed for osteoporosis at ages of 50,60,70, and 80 years is $13 \%$, $27 \%, 47 \%$, and $67 \%$ respectively (4). Fracture incidence especially hip fractures also increase progressively both in white women and elderly people (after age 70). Approximately $32 \%$ of women at age 80 years have hip fractures and $42 \%$ of women at advanced age have vertebral fractures $(5,6)$. In women, a rapid rise of vertebral fractures is usually related to the beginning of menopause and this fracture frequently followed by increasing of wrist and hip fractures.

Although the problem of osteoporosis in men is often less than that of women because the beginning and development of bone loss is slower in men; however, it is as an important public health problem in men too. All fractures can happen in men with osteoporosis. Approximately $33 \%$ of hip fractures are related to men every year and one-third of men with hip fractures will die before a year. With increasing age, hip fracture increases especially after age of 70 and this fracture is seen in $17 \%$ of 


\section{Implication for health policy/practice/research/ medical education}

Since people with osteoporosis are at high risk of fractures, therefore patients and health care providers should be concern about this disease. Health care providers should make individuals aware of detrimental and long terms effects of osteoporosis and give them beneficial guidelines to prevent or treat osteoporosis. Health care providers suggest many different approaches for preventing and treating of osteoporosis including exercise, healthful lifestyle, calcium and vitamin D intakes, and osteoporosis medicines. Some trials demonstrated that calcium supplementation intake does not have useful effects on fracture risk in postmenopausal women. Conversely, some studies encourage individuals to take calcium for decreasing osteoporosis and fracture risk.

men at age of $80(5,6)$.

\section{Pathogenesis}

Extreme bone resorption - that is followed by diminished bone mass - and the lack of bone formation during bone remodeling are major factors related to skeletal fragility. Therefore, knowing the process of bone remodeling (the most significant action of bone cells) is very important. Bone remodeling is done in two stages; the first stage is reversal or resorbtion and the second stage is osteoblastic replacement. In resorption stage, which is shorter than osteoblastic replacement phase of bone remodeling, old bone is removed and in osteoblastic replacement stage, old bone is replaced by new bone (7). Any factor that increases the rate of bone remodeling can decrease bone mass and increase bone fractures. Many factors are responsible for regulating of remodeling rates, including estrogens, androgens, vitamin $\mathrm{D}$, and parathyroid hormone (PTH), cytokines (various growth factors, interleukins, prostaglandins and tumor necrosis factor) $(8,9)$. However, the loss of bone rates are affected by genetic, endocrine, dietary factors, particularly calcium and vitamin $\mathrm{D}$, environmental factors (smoking, chronic diseases and medications and alcohol intake), menopause and aging and physical activity.

\section{Menopause}

Studies showed that bone resorption and bone formation are speeded up both at the menopause and at the premenopausal women. A decrease in ovarian activity at premenopausal women and in estrogen levels naturally at menopausal women can cause imbalance between bone formation and bone resorption by increasing osteoclastic resorption more than osteoblastic formation. This process causes osteoclasts, perforate bony trabeculae, while osteoblasts are not able to rebuild bone. This phenomena promotes osteoporosis in menopausal state (10).

\section{Aging}

With aging, imbalance between formation and resorption in both sexes increases in both sexes. Age-related bone loss is connected to both endogenous estrogen production and reduced renal calcium conservation efficiency (11).

\section{Dietary factors}

Calcium and vitamin D are two significant factors that contribute to most metabolic processes. Vitamin D is synthesized by humans and plants after exposure to sunlight and is taken from diet. Vitamin D goes through two-step hydroxylation. The first hydroxylation is occurred in the liver and the second hydroxylation is occurred in the kidneys. 25(OH) D is made in liver and then it is converted into 1,25-dihydroxyvitamin $\mathrm{D}(1,25[\mathrm{OH}] 2 \mathrm{D})$ in kidneys. Calcitriol has direct effects on the skeleton by stimulating calcium absorption. 25(OH)D levels is the most common blood indicator for measuring vitamin $\mathrm{D}$ in a person. Since vitamin $\mathrm{D}$ has an important role in blood regulation of phosphorous and calcium, therefore it can be suitable for some conditions caused by low levels of phosphorous and calcium. Vitamin D deficiency or insufficiency (less than $20 \mathrm{ng} / \mathrm{mL}$ ) can cause low bone mass, muscle weakness, and increase risk of osteoporosis and fractures in older adults $(11,12)$. Calcium intake, absorption, sweat, respiratory, and urinary excretion can specify the balance of blood calcium. About $30 \%$ of calcium received will absorb. Low calcium absorption may increase risk of fractures. Many factors have important effects on calcium absorption and excretion. Phytates, fiber, oxalates, iron can decrease calcium absorption (13). Extreme sodium and protein intake with low calcium intake increase urinary calcium excretion. Calcium intake, either alone or in combination with vitamin $\mathrm{D}$, can decrease bone loss in postmenopausal women and the risk of nonspine fractures (14). In elderly individuals, high protein and vitamin A intakes have been related to the risk of hip fractures. In addition, low phosphate and magnesium intakes may contribute to bone loss and increased fracture risk.

\section{Alcoholism}

Some studies have demonstrated that low alcohol intakes can increase bone mass. Conversely, high alcohol intakes can decrease osteoblastic replacement phase of bone remodeling and increase the risk of osteoporosis (15).

\section{Smoking and physical activity}

Smoking and physical activity are two important factors that have effects on bone mass. Smoking contributes to osteoblast malfunctions and earlier menopause (16). Therefore, smokers usually have low bone mass and are at high risk of fracture (16). In addition, resistance exercise has a positive effect on bone mass and also can decrease risk of fractures.

\section{Symptoms}

Osteoporosis is known as an asymptomatic disease in which person cannot feel any signs in the early stages of bone loss. But with aging, bones lose their strength and the risk of bone fractures increases. Therefore, patients will be aware of their osteoporosis when they experience any pain caused by fractures in the advanced stages of the disease. 
The most important and common signs of osteoporosis include fractures, back pain, loss of height and hunched posture. Most locations affected by fractures are hip, spine (vertebra) and wrist bones. Fractures usually happen as a result of minor activities that would not normally harm the individuals, such as slip-and-fall, walking, climbing stairs, and even sneezing or coughing. Osteoporosis makes bones thin and fragile (17). The brittle bones can collapse and break during minor activities. These compaction fractures can cause some symptoms such as pain, loss of height, and hunched-back posture of the upper back (kyphosis). Since kyphosis makes airway endures extra pressure therefore it affects breathing (18).

\section{Risk factors and causes}

Osteoporosis as a common bone disease may occur without any known cause but most of the time there are some factors that either cause bone loss or increase the risk of bone loss. Some of these risk factors are unchangeable. For example, elderly women who are white are the most likely to have osteoporosis and also people who have parents experienced osteoporosis have a higher risk. Other factors are related to life style. If individuals do not take enough calcium and if patient has an eating disorder or gastric bypass surgery that the body is not able to absorb enough calcium, then, the subjects are at high risk of osteoporosis. Low levels of calcium decrease bone density and increase risk of fractures. Osteoporosis is also associated with hormone levels in the body. Decreased estrogen levels at the time of menopause and increased thyroid hormone levels can develop osteoporosis (19). Other overactive glands such as parathyroid and adrenal glands can also make brittle bones. The use of certain medications for a long time, such as corticosteroid medications and medicines taken for cancer treatments (breast cancer in women and prostate cancer in men) can decrease hormone levels in patients. Estrogen levels in women can also diminish at the time of menopause and testosterone levels in men can also diminish as they age (20). Decrease of these hormone levels in people can develop osteoporosis. Other risk factors are related to your lifestyle, such as consuming a large amount of alcohol, smoking, sitting for a long time or not being active.

\section{Diagnose of osteoporosis}

Although people at age above 60-year-old are at high risk of osteoporosis, but it is possible to be seen low bone mass (osteopenia) or osteoporosis at earlier age. Since osteoporosis is known as an asymptomatic disease, individuals should be aware of any risk factors and follow an appropriate lifestyle and treatment tactics in consultation with a doctor. The major goal of diagnosing patients for osteoporosis is evaluating low bone mass, assigning recent fractures, and finally determining some steps to prevent or treat. There are many different types of bone mineral density (BMD) tests. These tests like simple bones (usually spine or hip) x-ray are done when your osteoporosis happens with aging. Simple bones x-ray can measure bone density, demonstrate fractures and predict the risk of future fractures and osteoporosis. The most common and important test is DXA (dual-energy $\mathrm{X}$-ray absorptiometry) (21). DXA is a test like X-ray with a low-radiation that is used to measure low percentages of bone density at the spine and hip or total body in both premenopausal and postmenopausal women. According the World Health Organization (WHO) osteoporosis in postmenopausal women is defined as a BMD value at the spine, hip, or forearm of 2.5 or more standard deviations (SDs) below the young adult mean (T-score $\leq-2.5$ ). Other BMD tests such as QCT (quantitative computed tomography) is used to measure the spine or hip, PQCT (peripheral QCT) is used to measure the forearm, QUS (quantitative ultrasound) is used to measure the finger or heel by using waves of sound, SXA (single-energy x-ray absorptiometry) is used to measure the wrist or heel, DPA (dual photon absorptiometry) is used to measure the spine, hip or total body, and SPA (single photon absorptiometry) is used to measure the wrist. There are many other methods such as blood and urine tests that identify both the metabolism of bone and osteoporosis occurred with medical condition (22).

\section{Prevention and treatment}

There are some important tips for prevention of osteoporosis in men and postmenopausal women and premenopausal. These tips are associated with dietary and lifestyle.

In men, fundamentally they must get enough calcium $(1000 \mathrm{mg} / \mathrm{d}$ in younger men, 1000 to $1200 \mathrm{mg}$ daily in older men, total diet plus supplement) and vitamin D (600 to 800 international units/day) supplementation. They also should stop smoking and drinking a lot of alcohol. Regular exercise and some hormonal medicines are effective in prevention of fractures and osteoporosis in men.

In postmenopausal women, the approach to treatment of osteoporosis is changing lifestyle and taking some medications. If these women want to change their lifestyle, they should take enough calcium and vitamin $\mathrm{D}$, exercise, stop smoking, and avoid drinking heavy alcohol. Institute of Medicine dietary reference intakes recommended $1200 \mathrm{mg}$ of calcium and 800 international units (IU) of vitamin D daily for postmenopausal women with osteoporosis (23). Oral bisphosphonates and IV bisphosphonates (for patients cannot tolerate oral bisphosphonates) are suggested as initial pharmacologic treatment. Bisphosphonates like alendronate, risedronate, ibandronate, and zoledronic acid can improve bone mineral density (BMD) and decrease the risk of fractures in postmenopausal women. Since estrogen has a protective effect on bone and the heart, it is also prescribed for osteoporosis and coronary heart disease (CHD). PTH and calcitriol are two significant hormones that modulate calcium and phosphate concentrations in serum. Within a narrow range of calcium, PTH can increase blood level of calcium by stimulating renal reabsorption and bone 
resorption. Therefore, exogenous PTH will be an effective hormone for the treatment of osteoporosis (19).

\section{Conclusion}

Since people with osteoporosis are at high risk of fractures, therefore patients and health care providers should be concern about this disease. Health care providers should make individuals aware of detrimental and long terms effects of osteoporosis and give them beneficial guidelines to prevent or treat osteoporosis. Health care providers suggest many different approaches for preventing and treating of osteoporosis including exercise, healthful lifestyle, calcium and vitamin D intakes, and osteoporosis medicines. Some trials demonstrated that calcium supplementation intake does not have useful effects on fracture risk in postmenopausal women. Conversely, some studies encourage individuals to take calcium for decreasing osteoporosis and fracture risk.

\section{Authors' contribution}

SB searched and gathered the related articles as well as writing. SB prepared the draft. HN edited the final manuscript several times. All authors read and signed the final paper.

\section{Conflicts of interest}

The authors like to declare that $\mathrm{HM}$ and $\mathrm{SB}$ are the current staffs of the journal. The peer-review process of the current article has been performed in accordance with the COPE guidelines.

\section{Ethical consideration}

Ethical issues (including plagiarism, data fabrication, double publication) have been completely observed by the authors.

\section{Funding/Support}

None.

\section{References}

1. Nordin BEC, Need AG. How can we prevent osteoporosis? In: Christiansen C, Johansen JS, Riis BJ, (eds). Osteoporosis. Copenhagen, Norhaven A/S; 1987:1204-10.

2. Ray N, Chan JK, Thamer M, Melton LJ. Medical expenditures for the treatment of osteoporotic fractures in the United States in 1995. J Bone Miner Res. 1997;12:24-35.

3. Cummings SR, Black DM, Thompson DE, Applegate WB, Barrett-Connor E, Musliner TA, et al. Effect of alendronate on risk of fracture in women with low bone mineral density but without vertebral fractures. JAMA. 1998;280:2077-82.

4. Looker AC, Orwoll ES, Johnston CC Jr, Lindsay RL, Wahner HW, Dunn WL, et al. Prevalence of low femoral bone density in older U.S. adults from NHANES III. J Bone Miner Res. 1997;12:1761-8.

5. Gallagher JC, Melton LJ, Riggs BL, Bergstrath E. Epidemiology of fractures of the proximal femur in Rochester, Minnesota. Clin Orthop. 1980;150:163-71.
6. Melton LJ, Kan SH, Frye MA, Wahner HW, O’Fallon WM, Riggs BL. Epidemiology of vertebral fractures in women. Am J Epidemiol. 1989;129:1000-11.

7. Cole RE. Normal bone remodeling process. Osteoporosis: Unmasking a Silent Thief. Brooklyn, Michigan: Wellpower Publications; 2000

8. Jilka RL, Hangoe G, Girasole G. Increased osteoclast development after estrogen loss: mediation by interleukin-6. Science. 1992;257:88-91.

9. Mundy GR, Boyce BF, Yoneda T. Cytokines and bone remodeling. Osteoporosis. San Diego, Calif: Academic Press; 1996:477-82.

10. Arts J, Kuiper GG, Janssen JM, Gustafsson JA, Löwik CW, Pols HA, et al. Differential expression of estrogen receptors and mRNA during differentiation of human osteoblast SVHFO cell. Endocrinology. 1997;138:5067-70.

11. Heaney RP. Vitamin D, nutritional deficiency, and the medical paradigm. J Clin Endocrinol Metab. 2003;88:51078.

12. Heaney RP. Calcium, parathyroid function, bone and aging. J Clin Endocrinol Metab. 1996;81:1697-8.

13. Heany RP. Calcium, dairy products and osteoporosis. J Am Coll Nutr. 2000;19:83S-99S

14. Reid IR, Ames RW, Evans MC, Gamble GD, Sharpe SJ. Long-term effects of calcium supplementation on bone loss and fractures in postmenopausal women: a randomized controlled trial. Am J Med. 1995;98:331-5.

15. Gonzalez-Calvín JL, Garcia-Sanchez A, Bellot V, MuñozTorres M, Raya-Alvarez E, Salvatierra-Rios D. Mineral metabolism, osteoblastic function and bone mass in chronic alcoholism. Alcohol Alcohol. 1993;28:571-579.

16. Grainge MJ, Coupland CA, Cliffe SJ, Chilvers CE, Hosking DJ. Cigarette smoking, alcohol and caffeine consumption, and bone mineral density in postmenopausal women. The Nottingham EPIC Study Group. Osteoporos Int. 1998;8:355-63.

17. Finsen V. Osteoporosis and back pain among the elderly Acta Med Scand. 1988;223:443-9.

18. Greendale GA, Barrett-Connor E, Ingles S, Haile R. Late physical and functional effects of osteoporotic fractures in women: The Rancho Bernardo Study. J Am Geriatr Soc. 1995;43:955-61.

19. Cranney, A, Guyatt, G, Griffith, L, Wells, G, Tugwell, P, Rosen C. Meta-analysis of therapies for postmenopausal osteoporosis, IX: summary of meta-analysis of therapies for postmenopausal osteoporosis. Endocr Rev. 2002;23:570-8.

20. Kurland ES, Cosman F, McMahon DJ, Rosen CJ, Lindsay R, Bilezikian JP. Parathyroid hormone as a therapy for idiopathic osteoporosis in men: effects on bone mineral density and bone markers. J Clin Endocrinol Metab. 2000;85:3069-76.

21. Kanis JA, Glüer C-C; Committee of Scientific Advisors, International Osteoporosis Foundation. An update on the diagnosis and assessment of osteoporosis with densitometry. Osteoporos Int. 2000;11:192-202

22. Marshall D, Johnell O, Wedel H. Meta-analysis of how well measures of bone mineral density predict occurrence of osteoporotic fractures. BMJ. 1996;312:1254-9.

23. Delmas PD. Treatment of postmenopausal osteoporosis. Lancet. 2002;359:2018-26. 